Benedikt George*, Michael Fink, Helmut Ermert, Pia T. Hiltl, Geoffrey Lee, and Stefan J. Rupitsch

\title{
Investigation of the Inertial Cavitation Activity of Sonosensitive and Biocompatible Nanoparticles for Drug Delivery Applications Employing High Intensity Focused Ultrasound
}

https://doi.org/10.1515/cdbme-2019-0147

\begin{abstract}
An approach to improve chemotherapy, while minimizing side effects, is a local drug release close to the tumorous tissue. For this purpose, the active drug component is often bound to nanoparticles employed as drug carriers. In the present study, we investigate sonosensitive, biocompatible poly-(L)-lactic acid (PLA) nanoparticles, which shall be used as drug carriers. For drug release, High Intensity Focused Ultrasound (HIFU) will be employed to introduce inertial cavitation, which separates the active drug component from the drug carrier. The cavitation effect generates an acoustic noise signal, which characterizes the cavitation activity and is expected to serve simultaneously as an indicator for the release of the active drug component. Depending on the ultrasound frequency, different acoustic levels of the inertial cavitation activity were measured. Investigations using a setup for passive cavitation detection (PCD) deliver quantitative results regarding the frequency dependence of the cavitation activity level of nanoparticles and reference media.
\end{abstract}

Keywords: Ultrasound, cavitation, nanoparticles, drug delivery.

\section{Introduction}

Several drug delivery systems have been investigated, which accumulate the drug close to the tumorous tissue.

One of these methods is Magnetic Drug Targeting (MDT), whereby the active drug component is bound to drug carriers consisting of iron oxide nanoparticles. They are guided to the cancerous tissue by means of a internal magnetic field gener-

*Corresponding author: Benedikt George, Department of Sensor Technology, Paul-Gordan-Street 3/5, Erlangen, Germany, e-mail: benedikt.george@fau.de

Michael Fink, Helmut Ermert, Stefan J. Rupitsch, Department of Sensor Technology, Paul-Gordan-Street 3/5, Erlangen,

Germany

Pia T. Hiltl, Geoffrey Lee, Department of Pharmaceutics, Cauerstraße 4, Erlangen, Germany ated by a strong extracorporal magnet. Though MDT was successfully tested in pre-clinical animal studies [1], this method is restricted to certain tumors only. The spatial decay of the magnetic field in the tissue limits the application to subcutaneous tumors. Moreover, active drug component release is not limited to the tissue to be medicated but it is initiated as soon as the drug is injected. The limited treatment depth as well as the spontaneous release of the drug can be avoided if the drug release needs to be enabled, for example by sonication of the drug carrying particles. In the following, we consider a drug delivery application, in which PLA nanoparticles are planned to serve as drug carrier and for which a High Intensity Focused Ultrasound (HIFU) transducer is employed for the cavitation exciting sonication.

The suspension consisting of nanoparticles, carrier liquid as well as an active drug component is intravenously injected near by the tumor. As opposed to healthy tissue, malignant tissue has a defective vascular system because of its rapid growth. It is therefore fenestrated, making it permeable to particles in the nanometer range. This effect is widely known as the Enhanced Permeability and Retention Effect (EPR). Local drug release is activated due to the sonosensitive behavior of the nanoparticles by the inertial cavitation effect, which is generated by a HIFU field irradiating the tumorous area from outside the body. The aim of this method is to keep the treatment time as short as possible and at the same time, to achieve a complete release of the active drug component. Presumably, the duration of the drug release depends on the cavitation activity, which is controlled by the emitted ultrasound signal.

Since the ultrasound signal is a high-energy mechanical wave leading to the inertial cavitation effect inside the body, tissue can become thermally and mechanically stressed. One way to avoid tissue hazards is to limit the mechanical index $M I$, which has been established by the US Department of Health and Human Services with a view to safe application of diagnostic ultrasound [2].

The mechanical index $M I$ is used to estimate possible mechanical damage to the biological tissue. Besides the nega- 
tive peak pressure $\hat{p}_{\text {neg }}$, the ultrasonic frequency $f$ is used to calculate the mechanical index $M I$

$$
M I=\frac{\frac{\hat{p}_{\mathrm{neg}}}{\mathrm{MPa}}}{\sqrt{\frac{f}{\mathrm{MHz}}}} .
$$

The negative pressure is decisive because in the negative pressure phase, cavitation nuclei are enlarged or rather vacuum bubbles are formed which subsequently implode and lead to inertial cavitation. Permissible values of the mechanical index for diagnostic ultrasound are in a range of about 1.9, depending on the tissue type. According to the $M I$, the tolerable pressure-dependent sound intensity $I(p)$ can be determined. In addition to the $M I$, the sonication time has to be considered, whereby an exposition of $50 \mathrm{Ws} \mathrm{cm}^{-2}$ should not be exceeded [3] to meet the permissibility of an diagnostic application of ultrasound

$$
I(p) \cdot t \leq 50 \mathrm{Ws} \mathrm{cm}^{-2} .
$$

The mathematical context described in eq. 2 is illustrated in Figure 1. Hereby, the green section marks the area in which no significant effects in biological tissue can occur, whereas the red lined section marks the area in which tissue injury due to medical ultrasound can appear. It is worth mentioning that ultrasound intensities above $3.5 \mathrm{~W} \mathrm{~cm}^{-2}$ are related to HIFU signals.

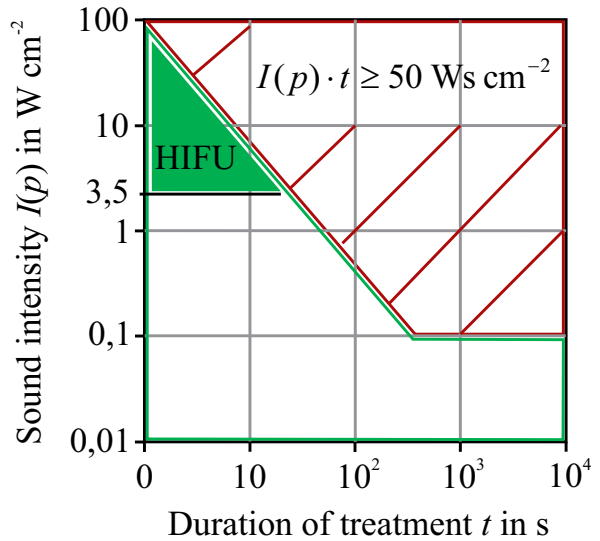

Fig. 1: Permissible and inadmissible range in ultrasound treatment.

For this reason, it is crucial to develop a suitable ultrasound signal defined by its signal amplitude, signal frequency and signal duration to induce inertial cavitation, taking the limit values described above into account. Within the scope of this work, the signal frequency dependent cavitation behavior in the frequency range between $500 \mathrm{kHz}$ and $1 \mathrm{MHz}$ is investigated.

\section{Experimental Setup}

\subsection{Biocompatible Nanoparticles}

The nanoparticles used for these investigations were produced at our Division of Pharmaceutics of the Friedrich-AlexanderUniversity (FAU) Erlangen-Nuremberg, Germany. So far, inertial cavitation could be stimulated on sonosensitive nanoparticles of suitable size at low ultrasonic frequencies only. However, low ultrasonic frequencies do not permit sufficient focusing limited to the desired tissue region. Recently, we designed new poly-L-lactid acid particles (spheres and capsules) with diameters around $120 \mathrm{~nm}$, which also show inertial cavitation at well focusable frequencies $(0.5-1 \mathrm{MHz})$. For the production process of the nanoparticles, an aqueous solution containing Poloxamer 188, trehalose, PLLA solution in dichlormethane, and acetone are mixed and then dried by means of a freeze-drying process, resulting in a cake [4]. It is assumed that the nanoparticles have rough surfaces resulting from the freeze-drying process, which lead to in small gas pockets acting as cavitation nuclei. Furthermore, it is supposed that the active agent adheres better to a rough surface. Before the cavitation activity is investigated, the PLA samples are rehydrated with degassed and deionized water.

\subsection{Measurement Setup and Experimental Procedure}

In order to explore an applicable ultrasound signal to instigate inertial cavitation for drug delivery applications, a passive cavitation detection (PCD) setup was used.

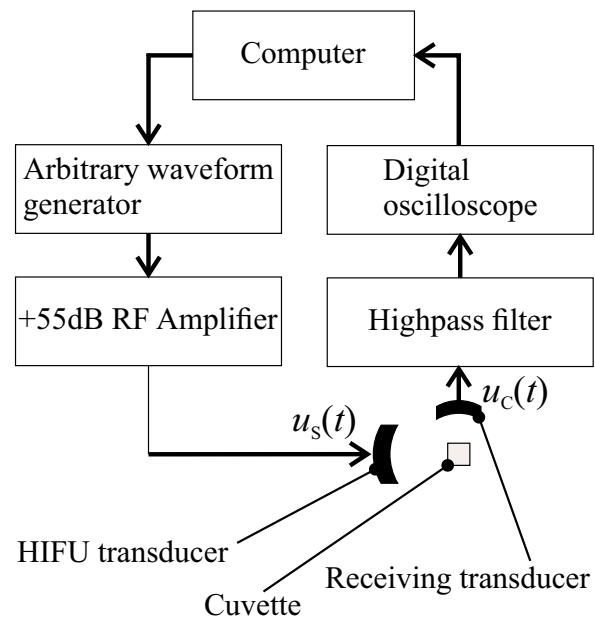

Fig. 2: Measurement setup to investigate inertial cavitation activity. 
The utilized PCD setup is shown in Fig. 2. The HIFU transducer (Sonic Concepts H231) is driven via an arbitrary waveform generator (Agilent $33522 \mathrm{~B}$ ) and an RF power amplifier (ENI-1140LA) is used to amplify the generated voltage signal $u_{\mathrm{S}}(t)$ by $55 \mathrm{~dB}$.

The nanoparticle suspension is filled in a polystyrene cuvette, which is mounted in a bracket. The cuvette and thereby the nanoparticles are placed in the focal volume of the spherically focusing HIFU transducer. By operating the transducer at its resonance frequency, a high sound energy is achieved in the focal volume [5].

The spherical shape of the transducer surface combined with the high ultrasonic frequency leads to a focused ultrasonic beam, whereby the sound energy is mainly concentrated in a focal volume shaped like an ellipsoid of about one millimeter lateral diameter and a few millimeters axial size. Outside the focal area, the sound pressure decreases by at least $-3 \mathrm{~dB}$. The small size of the focal volume is beneficial for medical applications, as the location of the active drug component release inside the tissue can be very accurately addressed with ultrasound.

To measure the cavitation activity signal of the PLAnanoparticles, a focused broadband ultrasonic transducer (Olympus Immersion Transducer V315-SU) is used, which is confocally positioned in a crossed beam orientation with respect to the HIFU beam axis. The experimental setup is placed in a water basin, which is filled with degassed pure water. The received high-frequency and broadband cavitation signal $u_{\mathrm{c}}(t)$ is filtered by a highpass filter to reduce unwanted crosscoupled frequency components of the transmitter signal. After filtering, the signal is measured with an digital oscilloscope (Agilent DSO7014B). Then, the signal is spectrally analyzed and the voltage spectral density (VSD) $S_{r m}$ is calculated - as described in [6] - in order to analyze the measurement results as well as to enable comparability of the results from subsequent measurements.

To excite inertial cavitation, we use a series of sinusoidal burst signals employing different frequencies of $550 \mathrm{kHz}$, $750 \mathrm{kHz}$ and $950 \mathrm{kHz}$, which are within the transducer's $3 \mathrm{~dB}$ bandwidth, whereas the burst length is left at $0.6 \mathrm{~ms}$ with a duty cycle of $0.02 \%$. Accordingly, the negative sound pressure is left at $340 \mathrm{kPa}$ for each burst signal that is regulated by matching the voltage amplitude at the function generator to the corresponding sound pressure level. This low negative sound pressure is safe for biological tissue since even for a small value of $M I=1$, the permissible negative sound pressures according to eq. 1 are in a range of $740 \mathrm{kPa}$ to $975 \mathrm{kPa}$ for the above mentioned frequencies. In addition, $340 \mathrm{kPa}$ corresponds to a sound intensity of $8 \mathrm{~W} \mathrm{~cm}^{-2}$ for a single burst and, for our application, to $0.02 \% \cdot 8 \mathrm{~W} \mathrm{~cm}^{-2}=1.6 \mathrm{~mW} \mathrm{~cm}^{-2}$ for the total burst sequence, which is far away from the critical powerdensity levels of diagnostic ultrasound.

It can be summarized that the ultrasound burst signal does not exceed the specified limits and can therefore be utilized for drug delivery applications.

\section{Measurement Results}

At first, we measured the cavitation activity of a positive and negative reference medium to classify the nanoparticles. A pharmaceutical product, Optison, serves as positive reference medium, which is usually utilized as a contrast agent in ultrasound imaging, particularly in echocardiography. It consists of a sterile suspension in which microspheres (gas bubbles) of Albumin filled with Perflutren with a diameter from $2.5 \mu \mathrm{m}$ to $4.5 \mu \mathrm{m}$ are dissolved. It is known that this pharmaceutical product shows cavitation due to HIFU excitation since the Perflutren gas bubbles implode. As a negative sample, pure water is appropriate. However, it must be ensured that the pure water does not contain any suspended particles and that it is degassed. Such impurities as well as air bubbles could serve as cavitation nuclei, which would falsify the measurement results.

Figure 3 illustrates the cavitation activity of Optison and pure water (1) in comparison to the PLA-nanoparticles (2) at the different frequencies. As already mentioned, pure water is a negative reference medium as it does not show any cavitation effect at the used frequencies. Though, the positive reference medium Optison shows a detectable cavitation activity. At excitation frequencies of $550 \mathrm{kHz}$ as well as at $750 \mathrm{kHz}$, Optison initially shows a strong inertial cavitation effect, which decreases by time as the number of gas bubbles gets reduced by cavitation. At the frequency of $950 \mathrm{kHz}$, the initial cavitation activity of Optison is halved and it is hardly present after a measurement period of $200 \mathrm{~s}$. The reason for this weak cavitation activity lies in a very short period of the negative pressure amplitude at high frequencies. Within this short negative pressure phase, only big perflutren bubbles exceed their critical size and are excited to cavitate.

Similar cavitation characteristics can be observed for the PLA-nanoparticles. In general, a higher VSD is detectable at lower frequencies. At the excitation frequency of $550 \mathrm{kHz}$, the VSD starts with a local maximum value and decreases continuously. The negative pressure phase is longest at this frequency. The cavitation bubbles can grow for a long time, so that the bubbles collapse will be very energetic and the cavitation will be accordingly strong. As a result, we assume that many cavitation nuclei are destroyed over time. However, this nuclei reduction yields a decreasing cavitation activity. At the 
medium frequency $(750 \mathrm{kHz})$, the cavitation effect is weaker, so the VSD decreases slower and remains almost constant after $300 \mathrm{~s}$. At $950 \mathrm{kHz}$, one notices that the nanoparticles tend to show no cavitation activity as the VSD is almost on the same level as for pure water.
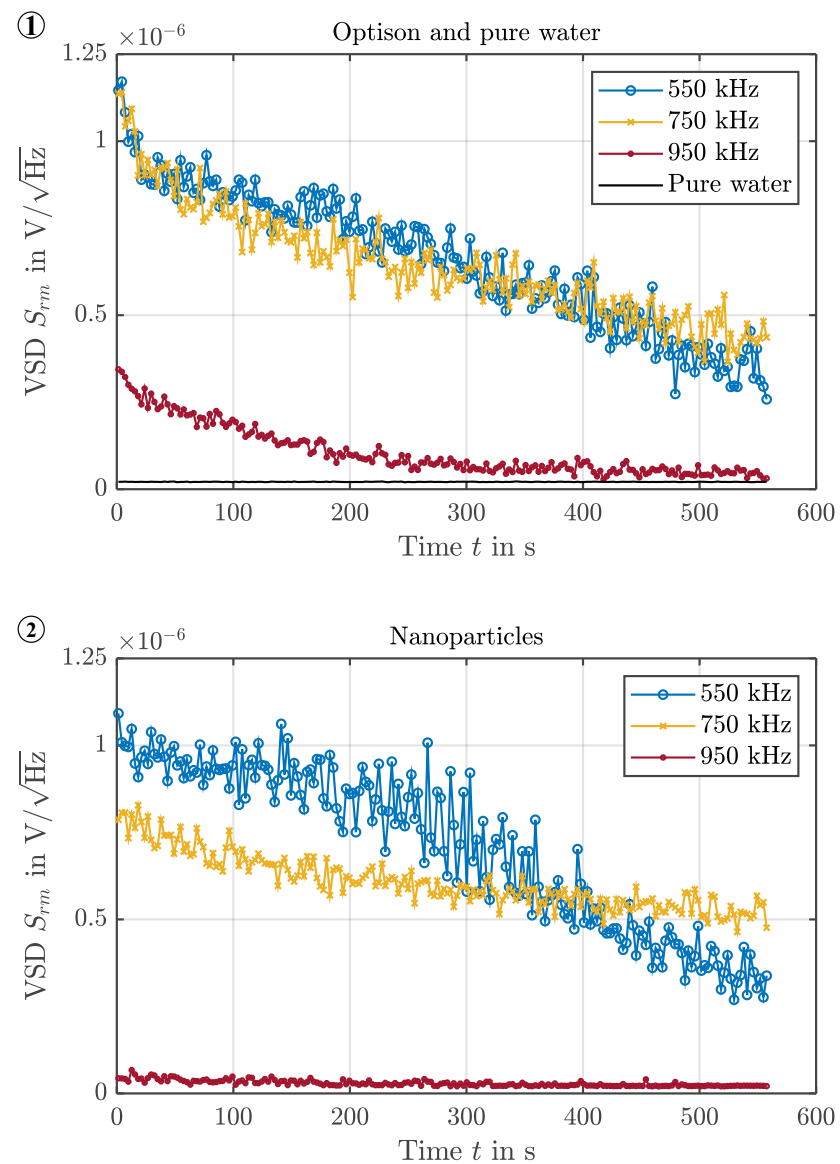

Fig. 3: Cavitation activity of Optison and pure water (1) compared to the nanoparticles (2) at different excitation frequencies.

Figure 4 summarizes the mean VSD of 10 samples from each test medium at the investigated frequencies. Apparently, the mean VSD of the nanoparticles is approximately equal to the VSD of Optison. For both media, the cavitation activity is low for $950 \mathrm{kHz}$ and almost on the same level for $550 \mathrm{kHz}$ and $750 \mathrm{kHz}$. As expected, pure water shows no cavitation activity at all.

\section{Conclusion}

In this study, we analyzed the cavitation activity of the nanoparticles at different ultrasonic excitation frequencies. Although the mean VSD of the cavitation activity is on the same

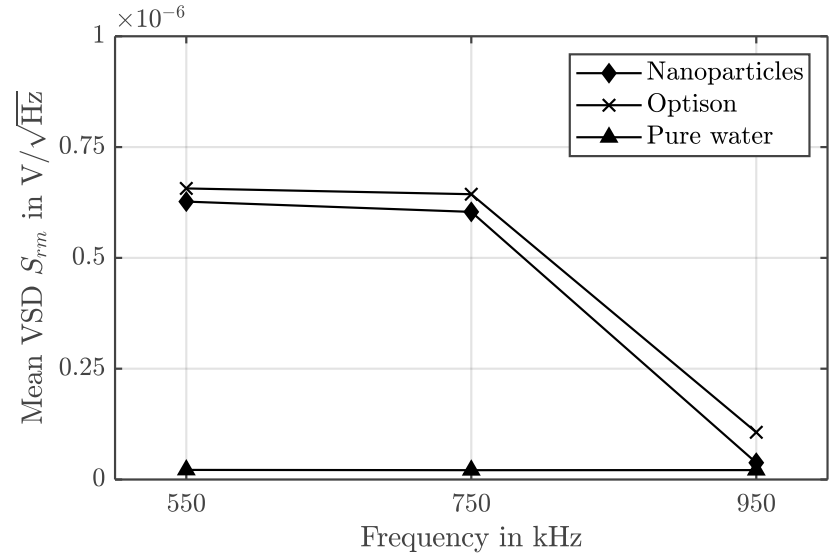

Fig. 4: Comparison of the mean VSD at different frequencies for the different fluids.

level for $550 \mathrm{kHz}$ and $750 \mathrm{kHz}$, we prefer $550 \mathrm{kHz}$ for further experiments since for this frequency, the initial cavitation activity is stronger, which could indicate a more efficient drug release. Future investigations will include other signal parameters (e.g. bandwidth, wave form, power) to design an optimal ultrasound signal for a high cavitation activity rate in order to enable an efficient localized drug release.

Acknowledgment: The authors gratefully acknowledge the financial support of the Deutsche Forschungsgemeinschaft (DFG) - project number ER 94/33-1, LE 626/16-1, RU 1656/21.

\section{References}

[1] Tietze R, Lyer S, Dürr S, Struffert T, Engelhorn T, Schwarz M, et al. Efficient drug-delivery using magnetic nanoparticlesbiodistribution and therapeutic effects in tumour bearing rabbits, Nanomed. 2013;9:961-971.

[2] Ermert H, Hansen C. Ultraschall. In: Dössel O, Buzug TM, editors. Biomedizinische Technik: Medizinische Bildgebung. 1st ed. Berlin: De Gruyter; 2014:217-314.

[3] Hill CR. Manual on health aspects exposure to non ionizing radiation. Chapter: Ultrasound. WHO Regional Office for Europe; 1977

[4] Hiltl PT, Hofmann A, Fink M, Rupitsch SJ, Lee G, Ermert H. Characterization of Acoustic Proberties of Poly(L)-lactic Acid Nanoparticles in terms of Inertial Cavitation, Proceedings of the 52nd Annual Conference of the German Society for Biomedical Engineering, Aachen 2018

[5] Rupitsch SJ. Piezoelectric Sensors and Actuators. Springer Berlin Heidelberg, 2019.

[6] George B, Fink M, Plock M, Hiltl PT, Ermert H, Lee G et al. Measurement of the Cavitation Activity of Sonosensitive Nanoparticles used for Local Chemotherapy, Proceedings of the 6. Tagung Innovation Messtechnik, Linz 2019. 